\title{
Gamificación combinada con aula invertida, aplicación en un grado de ingeniería.
}

\section{Gamification combined with flipped classroom, application in an engineering degree.}

\author{
Ricado Castedo $^{1}$, Javier Fernández-Torres ${ }^{1}$, Lina M. López ${ }^{1}$, María Chiquito ${ }^{1}$, Anastasio P. Santos ${ }^{1}$, José E. Ortiz ${ }^{1}$, \\ Ana P. Pérez-Fortes ${ }^{1}$, Marcelo F. Ortega ${ }^{2}$ \\ ricardo.castedo@upm.es, javier.fernandez.torres@alumnos.upm.es, lina.lopez@upm.es, maria.chiquito@upm.es, \\ tasio.santos@upm.es, joseeugenio.ortiz@upm.es, anapatricia.perez@upm.es, mf.ortega@upm.es \\ ${ }^{1}$ Departamento de Ingeniería Geológica y Minera \\ Universidad Politécnica de Madrid \\ Madrid, España \\ ${ }^{2}$ Departamento de Energía y Combustibles \\ Universidad Politécnica de Madrid \\ Madrid, España
}

\begin{abstract}
Resumen- Se ha desarrollado la metodología de aula invertida y gamificación en la asignatura obligatoria "Transferencia de Calor y Materia" de $2^{\circ}$ curso del grado de Ingeniero de la Energía en la ETSIME de la Universidad Politécnica de Madrid. Esta experiencia se realiza durante el curso 2018-19 en las dos clases en que se divide esta materia, considerados ambos como grupo experimental. El contraste de resultados se realiza con los grupos que cursaron la misma materia durante el 2017-18, donde "sólo" se tenía aula invertida y que se toman como el grupo de control. El material del que dispone el alumno en ambos casos es el mismo. A pesar de notarse un ligero descenso en la asistencia a clase, los resultados obtenidos por ambos grupos muestran que la inclusión de estas actividades supone un aumento de las notas de casi un punto en cada bloque. Asimismo, se analizan los resultados de los exámenes entre los alumnos que han tenido gamificación activa y los que no. En este caso la diferencia es aún mayor (en torno a 2 puntos en cada bloque). La dispersión en las notas se mantiene constante, estando ambos grupos igualmente nivelados.
\end{abstract}

Palabras clave: Aula invertida, gamificación, aprendizaje basado en problemas, tasas de asistencia, transferencia de calor y materia.

Abstract- The flipped classroom and gamification methodology has been applied to the compulsory subject "Heat and Matter Transfer", from the 2nd year of the Energy Engineering degree at the ETSIME at Universidad Politécnica de Madrid. This experience is carried out during the academic year 2018-19, where the subject is divided in two students' groups called experimental group. The results are compared with the students of the same subject from the academic year 20172018 that "only" had flipped classroom, and considered hereinafter as the control group. The material available for the students in both years is the same. Despite a slight decrease in class attendance, the results obtained by both groups show that the inclusion of both activities increases almost one point the general scores in each block. Likewise, the results of the examinations are analyzed between the students who participated in an active gamification and those who did not. In this case, the difference is even greater (around 2 points in each block). The scattering of the scores remains constant, having both groups a very similar level.

Keywords: Flipped classroom, gamification, problem-based learning, class attendance ratio, heat and mass transfer.

\section{INTRODUCCIÓN}

En el modelo de enseñanza tradicional, la información se dirige principalmente de profesor a alumno, con una interacción entre los actores implicados (profesor-alumno y alumnoalumno) muy escasa. En algunos casos, los profesores realizan ejercicios durante el desarrollo de las clases de forma que el estudiante, en oposición al clásico rol de receptores pasivos de información, participan de una forma más activa. A pesar de que algunos investigadores demuestran que una mayor participación del alumno implica un mejor rendimiento del mismo, muchos otros se encuentran reacios a nuevos métodos de aprendizaje con un carácter más participativo por parte del alumnado (Velegol et al., 2015). Algunas de las nuevas metodologías que buscan una mayor participación del alumno en clase surgen también como consecuencia de la introducción de los ordenadores en nuestra vida diaria, así como el aula invertida (Baker, 2000; Lage et al., 2000).

El aula invertida ha alcanzado una mayor visibilidad recientemente en diferentes áreas; a pesar de ello, esta metodología no ha adquirido tal aceptación en las asignaturas STEM (Science, Technology, Engineering, Mathematics; Ciencia, Tecnología, Ingeniería, Matemáticas) (Kerr, 2015). Posiblemente los primeros trabajos publicados fueron los de Bland (2006) y Gannod et al. (2008) en los grados de ingeniería eléctrica e ingeniería de software, respectivamente. En ambos casos se ha usado una primera versión de la metodología (sin implementación tecnológica), en la cual se propone a los alumnos ejercicios previos a la clase impartida por el profesor. Papadopoulos \& Santiago-Roman (2010) presentaron la aplicación de un aula invertida, donde el trabajo fuera de clase estaba basado en presentaciones de PowerPoint y ejercicios. Por el contrario, durante el desarrollo de las clases se basaba en discusiones y actividades basadas en este material. Los autores descubrieron que la nueva metodología era más efectiva que el curso tradicional. McClelland (2013) aplicó este método en alumnos de segundo año de la asignatura de mecánica de fluidos y comparó los resultados obtenidos con los del año anterior, que estudiaron con una metodología clásica. En este caso, los 
alumnos con aula invertida experimentaron unas notas estadísticamente significativas menores respecto a los otros estudiantes. Fidalgo-Blanco et al., 2017 encontró también una diferencia estadística en los resultados de una parte de un curso de fundamentos de programación usando una metodología llamada Micro flip teaching. Karabulut-Ilgu et al. (2018), demostró que las notas medias de alumnos que trabajaron con aula invertida es mayor que aquellos que estudiaron con una metodología tradicional, sin diferencia estadística, tras haber analizado 25 estudios diferentes. Junto con estas experiencias, el uso de la gamificación también ha mejorado los resultados académicos de los alumnos. Sirvan como ejemplo los trabajos de Hung (2016) y Yildirim (2017). Ambos autores descubren en sus estudios cómo la introducción de tecnologías de juego en el desarrollo de las clases supone una influencia positiva en el proceso de aprendizaje, en relación a los resultados, percepciones y experiencias. Ambos autores emplean la software Kahoot! como herramienta para tomar datos del proceso de gamificación.

Según diferentes autores (Hao, 2016; Karabulut-Ilgu et al., 2018), hay una clara necesidad de medir objetivamente el impacto del aula invertida en alumnos de grado en diferentes escenarios de aprendizaje, especialmente en STEM. En este trabajo se presentan los resultados obtenidos de la mezcla de aula invertida con técnicas de gamificación para una asignatura obligatoria de $2^{\circ}$ curso de ingeniería, tradicionalmente presencial pero con una alta carga de realización de problemas / ejercicios. El objetivo principal es detectar si la gamificación supone un refuerzo positivo al uso del aula invertida, ya contrastado en cursos anteriores (Castedo et al., 2017; Castedo et al., 2019).

\section{CONTEXTO}

En los primeros cursos de cualquier ingeniería, los alumnos vienen con una base académica, una predisposición y una actitud muy dispar. La finalidad fundamental de la metodología desarrollada y aplicada es mejorar el proceso de aprendizaje del alumnado y de algunas de sus competencias transversales. Para ello, decidimos emplear la técnica de aula invertida, combinada con otras actividades enlace basadas en cuestionarios y trabajos cooperativos en el aula donde el profesor se integra con los alumnos en la resolución de los problemas. Además, en ciertas sesiones los problemas se resuelven con la ayuda de cuestionarios implementados en Kahoot!.

Este trabajo se ha desarrollado en la asignatura "Transferencia de Calor y Materia", de la titulación Grado de Ingeniero de la Energía de la ETSI Minas y Energía de la Universidad Politécnica de Madrid durante el curso 18-19. La asignatura es parte del segundo semestre del segundo curso, consta de 6 créditos y es de carácter obligatorio.

La materia está dividida en 4 partes que, en orden cronológico, son: conducción (20 h - las 9 primeras clases), convección $(20 \mathrm{~h}$ - de la clase 10 a la 18$)$, radiación $(6 \mathrm{~h}$ - de la 19 a la 21) y transferencia de masa $(14 \mathrm{~h}$ - de la clase 22 a la 27). Entre paréntesis se muestran las horas de clase impartidas por el profesor en cada una de las partes. La asignatura comenzó en Febrero de 2019 y terminó en Mayo de 2019, siendo el examen final en Junio y el extraordinario en Julio de 2019. Todos los bloques tienen un examen liberatorio de evaluación continua. Los resultados que aquí se presentan y analizan corresponden a las partes más importantes de la materia: conducción, convección y transferencia de masa. Estas partes forman el $89 \%$ del contenido y, por ende, de la evaluación de la asignatura.

Este trabajo pretende analizar cómo afecta la implementación de la gamificación al desarrollo de las clases, las cuales se llevan a cabo mediante la metodología de aula invertida desde el curso 2016-17. Para ello, se compararán las calificaciones obtenidas por los alumnos que cursaron el año 2017-18 dicha asignatura (con aula invertida pero sin gamificación), con los del presente 2018-19 (con aula invertida y gamificación). De esta forma, quedarán establecidos el Grupo de Control y el Grupo Experimental, respectivamente (GC y GE de aquí en adelante).

En el presente curso (2018-19), se tienen dos grupos, el grupo 1 (G1) con 88 alumnos y el grupo 2 (G2) con 74 alumnos. Sin embargo, durante el curso anterior (2017-18), los dos grupos estaban divididos en 79 alumnos (G1) y 81 alumnos (G2).

\section{DESCRIPCIÓN}

La metodología, pese a ser impartida por varios profesores, tiene una implementación y aplicación en el aula muy similar, por lo que cabe esperar que los resultados sean similares en cada uno de los bloques. En la Figura 1 se puede observar un breve resumen de las actividades realizadas, que en detalle son:

\section{A. Test control de homogeneidad}

El segundo día de clase se lleva a cabo un test sencillo de 5 preguntas donde se pretende ver la comprensión de los alumnos acerca del material impartido durante la primera lección. Asimismo, dicho test servirá como punto de partida en nuestro estudio comparativo entre GE y GC, pues no tendría sentido la comparación en el caso de que el nivel inicial de los distintos grupos fuese radicalmente distinto.

\section{B. Recursos y tareas previas al aula con Moodle y Youtube}

Como parte de la metodología implantada en el curso 201617, y que se ha mantenido durante los siguientes cursos de estudio, se pone a disposición del estudiante el siguiente material:

- Libro de consulta en castellano (Sanchidrián, 2014) que incluye los temas de conducción y convección con ejercicios propuestos (disponible en pdf). Además, los alumnos disponen de apuntes realizados ad hoc para la parte de transferencia de masa, durante el curso 2016-17.

- Presentaciones de Power Point realizadas para cada sesión, las cuales sirven a modo de esquema y resumen.

- Videos explicativos de entre 5 y 10 minutos.

- Actividad enlace que consiste en un cuestionario de 5 preguntas, mezclando respuestas múltiples o verdadero/falso, obligatorio y por saturación. En estos cuestionarios, el alumno debe obtener un 10, teniendo la posibilidad de realizar múltiples intentos sin penalización, para poder acceder al material de la siguiente clase o clases, y así sucesivamente.

- Colección de problemas resueltos que los alumnos encuentran disponible al llegar a la última clase del bloque de la asignatura. 


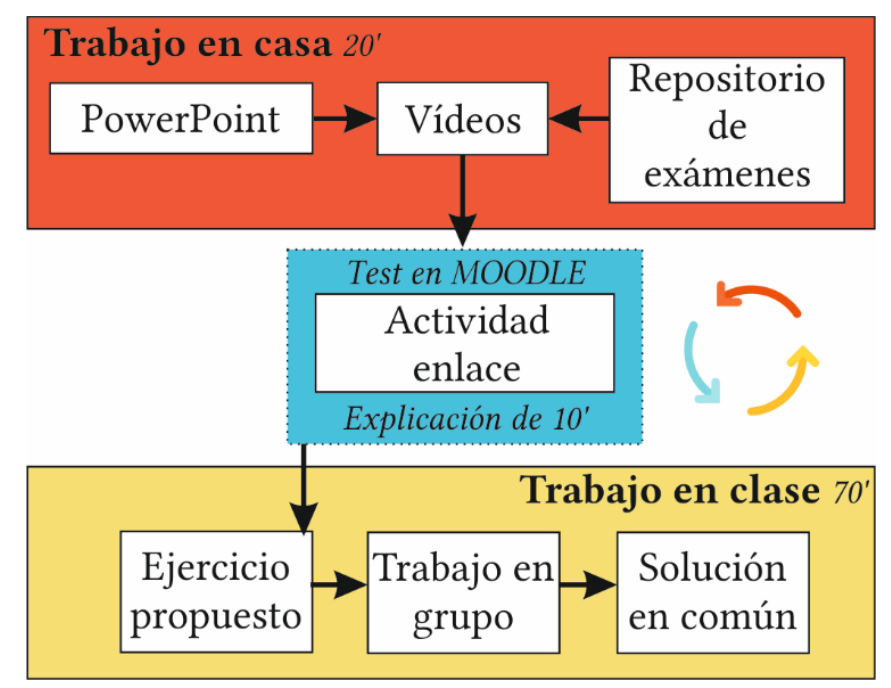

Figura 1. Esquema de la metodología empleada y la distribución de tiempos.

\section{Actividad en el aula}

En el aula los primeros 10 minutos se dedican al repaso de los resultados obtenidos en la actividad enlace, especialmente cuando dichos resultados son considerablemente malos para alguna de las cuestiones realizadas. Debido a que el alumno ya dispone de los conocimientos necesarios, se omite la explicación teórica característica de una enseñanza más tradicional y se dedicará la totalidad del tiempo a la resolución de problemas por equipos. En todos los bloques se plantean 2 problemas por clase. De esta forma, se logra dinamizar más la clase al mismo tiempo que se fomenta el trabajo en equipo, aptitud que les será esencial a los alumnos en su posterior carrera profesional.

Posteriormente, el profesor propone un ejercicio a los alumnos del aula, que trabajan en grupos de entre 3 y 5 personas. Son los propios alumnos los que eligen a sus compañeros de grupo. Durante este tiempo, el profesor sirve como comodín que ayuda a los alumnos en caso de dudas puntuales o dudas que no permitan seguir avanzando en el problema. Cuando estas dudas son generalizadas, el profesor explica en la pizarra lo necesario para ayudar a todos los grupos.

El trabajo en casa está relacionado con el posterior trabajo en clase mediante las actividades a realizar en Moodle, pues sólo estas darán acceso al contenido de las siguientes clases (ver Figura 1). Cada dos clases habrá un nuevo cuestionario, repitiéndose así el ciclo a lo largo del curso.

Durante el curso 2018-19, como novedad, algunos de los problemas resueltos en clase, alrededor de un tercio por bloque, se han resuelto empleando el software Kahoot! instalado en los móviles de los alumnos. Estos problemas se han resuelto de dos maneras diferentes:

- En algunos casos el Kahoot! se lanzaba al final del problema y los alumnos iban contestando a las preguntas planteadas, de tal manera que si habían resuelto de manera satisfactoria el ejercicio, se colocaban en los primeros puestos del ranking.

- En otros casos, el Kahoot! se lanzaba a medida que el problema se iba resolviendo por todos los miembros de la clase, siendo ésta una forma de trabajar la inteligencia colectiva. Así, se da la oportunidad a todos los alumnos de ir reenganchándose al juego de resolver el problema y así tener la posibilidad de obtener puntos extra.

Se establece un ranking en cada bloque de la asignatura, la forma de puntuar en dicho ranking es (Figura 2): en los ejercicios con Kahoot! se dan 3 puntos a los dos primeros alumnos en responder correctamente, 2 puntos a los que terminan en tercera y cuarta posición, y 1 punto al que termina quinto; en los problemas sin uso del Kahoot!, se da un punto a los alumnos del grupo o grupos que terminan primero el problema, y dos puntos al alumno que sale a la pizarra a explicar el resultado. Al final del bloque se evalúan los puntos obtenidos y se establece un sistema de premios finales por bloque. Se otorgará 1 punto extra (respecto a la nota del examen de dicha parte) a los 3 mejores, y 0.5 puntos a los siguientes 4 alumnos que encabecen la lista. De esta forma, se pretende incentivar al alumno para que estudie día a día y mejorar el rendimiento general de la clase.

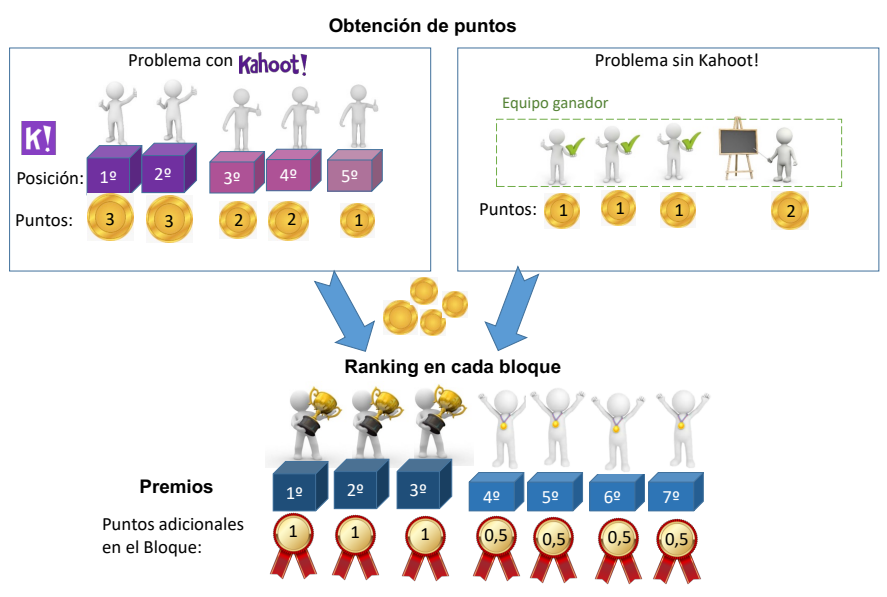

Figura 2. Distribución de los puntos por problema, y distribución final por bloque.

\section{Examen de evaluación}

Por último, pero no menos importante, tenemos la evaluación. Ésta se realiza en base a exámenes parciales liberatorios y compensatorios. Todo alumno que tenga más de un 3 en un examen, puede no presentarse al final siempre que la media ponderada con el resto de partes dé como resultado un aprobado ( $>5$ sobre 10). Los resultados aquí presentados se basan en los exámenes de conducción, convección y transferencia de masa:

- Conducción: el examen consta de tres preguntas teóricoprácticas donde el alumno puede llevar un resumen en papel de hasta tres hojas de lo que considere importante, sin ejercicios.

- Convección: el examen consta de un problema, donde el alumno puede llevar todo lo que estime oportuno, incluidos libros y ejercicios, siempre que sea en formato papel.

- Transferencia de masa: la estructura de este examen es la misma que la aplicada en el bloque de conducción. Pero en este bloque el alumno puede llevar las tres hojas de conducción, y otras tres realizadas para este bloque.

\section{Resultados}

El primer resultado es el del test de control de homogeneidad entre los grupos de los cursos 2017-18 y 2018-19. Los 
resultados se corresponden con el test de 5 preguntas relacionadas con lo enseñado en la clase 1 y donde sólo se consideran las respuestas correctas. Para la comparación de los resultados se emplea la prueba no paramétrica de U de MannWhitney (UMW de aquí en adelante) con el objetivo de ver si se puede o no se rechazar la hipótesis nula que supone que las dos muestras, las notas de G1 y G2 de cada curso, de distribuciones continuas tienen la misma mediana para un valor de significancia de 0,05. En ambos casos, los valores son superiores al $0,05 \mathrm{y}$, por tanto, no se puede rechazar que las distribuciones seas distintas. Esto implica que, los grupos de cada curso se pueden mezclar y, por ende, tenemos un grupo único para el curso 2017-18 y que llamaremos grupo de control (GC); y un grupo único para el curso 2018-19 y que llamaremos grupo experimental (GE).

Comparando con el UMW, el p-valor obtenido para dicho test entre GC y GE es de 0,180. Al ser mayor que la significancia elegida, no se puede rechazar que las distribuciones y, por lo tanto, el nivel de los alumnos, sea distinto. Ello a su vez implica que cualquier variación podría ser relacionada a la metodología empleada.

Una vez visto que los grupos sí se pueden comparar entre sí, resulta de interés analizar la asistencia a clase. Tal y como se observa en la Figura 3, vemos que hay cierta tendencia a la baja en lo que a porcentaje a asistencia se refiere, aunque la variación tanto para conducción como para convección es mínima (en torno al 5\%). Cabe destacar una variación brusca en el bloque de masa, donde el GE sufre una caída considerable de asistencia, tanto respecto al GC como respecto al mismo grupo en otros bloques.

En cuanto a la caída general en asistencia en todos los bloques, podemos encontrar distintas causas, aunque la más razonable es la pérdida de estímulo por parte del alumno al ver que los puntos de gamificación sufren muy pocas variaciones y los puntos extra suelen ir siempre a las mismas personas. Esto es una limitación de la gamificación donde un exceso de competición, puede desmotivar a los alumnos que no ganan. Asimismo, cabe destacar que, durante el curso 2018-19, el bloque de masa se realizó el último (en mayo), mientras que en el curso anterior se realizó en abril. Y debido que la asistencia a clase va decreciendo a lo largo de cualquier curso el hecho de que este año se haya impartido con posterioridad ha sido clave en este fenómeno.

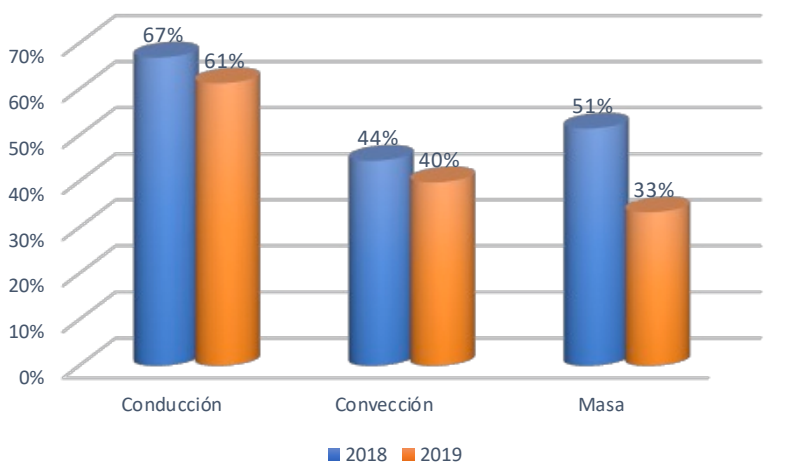

Figura 3. Comparación de la asistencia entre GC (2018) y GE (2019) para cada uno de los bloques. La asistencia se ha calculado como la media de asistentes a cada una de las sesiones del bloque.
Ahora bien, el siguiente paso sería analizar las notas obtenidas en cada uno de los bloques mediante dos comparativas, entre GE y GC; y entre los alumnos de GE que han obtenido bonus respecto a los que no (sin tener en cuenta obviamente la nota extra, sólo la nota del examen). Para cada uno de los bloques se han realizado los tests de UMW y la prueba de Kolgomorov Smirnov (KS de aquí en adelante). Esta última servirá para analizar si ambas muestras vienen de la misma distribución o no. Para ambos se emplea un nivel de significación de 0,05.

Tal y como se puede ver en la Tabla 1, siendo similar el porcentaje de alumnos presentados respecto a los matriculados, el porcentaje de aprobados ha aumentado drásticamente tanto en convección como en masa. En conducción, sin embargo, el porcentaje decreció muy ligeramente. A excepción de masa, donde las notas en el GE fueron significativamente muy superiores (la media aumentó en un punto), los valores obtenidos para los tests estadísticos también denotan un valor estadísticamente diferente, a pesar de tener una menor tasa de presentados. De este modo, podemos notar cómo la inclusión de la gamificación al aula invertida supone un estímulo añadido al alumnado, el cual se ve reflejado en sus resultados.

Tabla 1. Comparativa de las notas en cada uno de los bloques entre GE y GC. Notar que P/M es el ratio entre alumnos presentados y matriculados, y $\mathrm{A} / \mathrm{M}$ es el ratio entre alumnos aprobados y presentados.

\begin{tabular}{lcccccc}
\cline { 2 - 7 } & \multicolumn{2}{c}{ Conducción } & \multicolumn{2}{c}{ Convección } & \multicolumn{2}{c}{ Masa } \\
\cline { 2 - 7 } & GE & GC & GE & GC & GE & GC \\
\hline Media & 5,95 & 5,85 & 4,04 & 3,79 & 5,34 & 4,44 \\
\hline Desv. Típica & 2,72 & 2,79 & 2,58 & 1,75 & 2,58 & 2,71 \\
\hline Mediana & 6,50 & 5,90 & 4,10 & 3,90 & 5,30 & 4,40 \\
\hline P/M (\%) & 91 & 89 & 83 & 82 & 78 & 86 \\
\hline A/P (\%) & 69 & 71 & 43 & 26 & 63 & 44 \\
\hline $\begin{array}{l}\text { p-valor } \\
\text { (UMW) }\end{array}$ & \multirow{2}{*}{0,756} & 0,478 & \multirow{2}{*}{0,006} \\
\hline p-valor (KS) & \multicolumn{2}{c}{0,469} & 0,140 & \multicolumn{2}{c}{0,038} \\
\hline
\end{tabular}

Por otro lado, se ha realizado la comparativa entre los alumnos que sí han obtenido nota extra respecto a los que no. En este caso, la diferencia se hace aún más notable, tal y como se ve reflejado en la Tabla 2. En dicha tabla, se comparan las notas de los exámenes de los alumnos que han tenido puntos extra por gamificación (obviamente en esta comparativa esos puntos no se tienen en cuenta), con los que no la han obtenido. Este análisis se basa en la comparativa de los alumnos que han trabajado más y mejor durante el curso en las clases, con los que no. El resultado, nada sorprendente, es que la nota media de los alumnos que han trabajado regularmente y han obtenido esa gamificación, es de hasta dos puntos más que sus compañeros.

Los resultados obtenidos, son estadísticamente significativos, basándonos en los p-valores en dos de los tres bloques. Este fenómeno se puede deber a que los alumnos que obtienen estos extras son aquellos que más estudian día a día. Es, por tanto, un indicador del grado de esfuerzo de unos alumnos frente a otros. Todo ello se ve bien reflejado en aquellos exámenes donde las notas son inferiores. Por ejemplo, en el bloque de masa, la media de alumnos sin bonus fue inferior al aprobado $(4,95)$, mientras que los alumnos que más han trabajado obtuvieron una nota media muy superior $(7,75)$. En este caso, además, las desviaciones típicas son más bajas en los alumnos con puntos extra en los bloques, lo que significa un 
mayor nivel medio del alumnado puesto que está mejor preparado.

Tabla2. Comparativa entre los alumnos que han obtenido nota extra por su rendimiento en clase (NE en la tabla) y los que no $(\mathrm{N})$.

\begin{tabular}{lcccccc}
\cline { 2 - 7 } & \multicolumn{2}{c}{ Conducción } & \multicolumn{2}{c}{ Convección } & \multicolumn{2}{c}{ Masa } \\
\cline { 2 - 7 } & $\mathrm{NE}$ & $\mathrm{N}$ & $\mathrm{NE}$ & $\mathrm{N}$ & $\mathrm{NE}$ & $\mathrm{N}$ \\
\hline Media & 7,60 & 5,71 & 5,90 & 3,84 & 7,75 & 4,95 \\
\hline Desv. Típica & 1,96 & 2,74 & 2,75 & 2,52 & 2,24 & 2,42 \\
\hline Mediana & 7,50 & 6,00 & 5,70 & 3,50 & 8,20 & 5,00 \\
\hline $\begin{array}{l}\text { p-valor } \\
\text { (UMW) }\end{array}$ & \multirow{2}{*}{0,004} & 0,112 & 0,001 \\
\hline p-valor (KS) & 0,043 & 0,502 & 0,013 \\
\hline
\end{tabular}

\section{CONCLUSIONES}

Hoy en día, las escuelas de ingeniería deben producir graduados que sean capaces de resolver problemas, de aprender por sí mismos y que tengan un buen conocimiento técnico. Es aquí donde tanto el aula invertida como la gamificación resultan esenciales, y especialmente útiles si se combinan. La introducción de estas actividades, supone un valor añadido, no sólo a las notas del alumno a lo largo de su grado académico, sino que también supondrá una mejora en determinadas competencias transversales como el trabajo en equipo o el trabajo autónomo.

Tras haber analizado detalladamente los resultados obtenidos, hemos visto los efectos producidos por la aplicación de esta metodología:

- la gamificación premia al alumno que realiza un trabajo constante, aunque puede desmotivar al alumno que no lo hace,

- las notas con la inclusión de la gamificación, son sistemáticamente más altas,

- $\quad$ el porcentaje de aprobados frente a presentados, es también más alto si incluimos gamificación en aula invertida,

- $\quad$ sin embargo, el uso de la gamificación no ha paliado el descenso en asistencia a las clases.

Los cuestionarios y todo el material nuevo que el profesorado tenga que elaborar se pueden utilizar sin apenas ninguna modificación a largo plazo. Por tanto, la sostenibilidad de esta metodología es igual de simple que la de cualquier otro método de enseñanza menos participativo. Sin embargo, esta metodología sufre de ciertas limitaciones. Debido al tipo de actividades que exige, su aplicación en aquellas asignaturas principalmente teóricas se antoja difícil, si no en muchos casos imposible. Las asignaturas STEM, sin embargo, suelen basarse en la resolución de problemas, siendo su uso en dicho caso idónea.

\section{AgradeCIMIENTOS}

Los autores quieren agradecer a la Universidad Politécnica de Madrid (UPM) la financiación aportada a través del proyecto "Gamifiquemos para motivar: integración con inteligencia colectiva y aula invertida" con código IE1819.0606 de la convocatoria competitiva "Ayudas a la innovación educativa y a la mejora de la calidad de la enseñanza - 2019" de la UPM.
Baker, J. W. (2000). The “classroom flip": Using web course management tools to become the guide by the side. Paper presented at the 11 th International Conference on College Teaching and Learning, Jacksonville, FL.

Bland, L. (2006). Applying flip/inverted classroom model in electrical engineering to establish life-long learning. Paper presented at the annual meeting of the American Society for Engineering Education, Chicago, IL.

Castedo, R., López, L. M., Chiquito, M., Navarro, J., Cabrera, J. D., \& Ortega, M. F. (2019). Flipped classroomcomparative case study in engineering higher education. Computer Applications in Engineering Education, 27(1), 206-216.

Castedo, R., López, L. M., Ortega, M. F., Cabrera, J. D., GarcíaMartínez, M. J., Sanchidrián, J. A., Segarra, P. \& Paredes, C. (2017). Aula invertida para la mejora del aprendizaje en la asignatura de Transferencia de Calor y Materia. En IV International Conference on Learning, Innovation and Competitiveness--CINAIC.

Fidalgo-Blanco, A., Martinez-Nuñez, M., Borrás-Gene, O., \& Sanchez-Medina, J. J. (2017). Micro flip teaching-An innovative model to promote the active involvement of students. Computers in Human Behavior, 72, 713-723.

Gannod, G. C., Burge, J. E., \& Helmick, M. T. (2008). Using the inverted classroom to teach software engineering. Proceedings of the Annual International Conference on Software Engineering. Leipzig, Germany.

Hao, Y. (2016). Exploring undergraduates' perspectives and flipped learning readiness in their flipped classrooms. Computers in Human Behavior, 59, 82-92.

Hung, H. T. (2016). Clickers in the flipped classroom: bring your own device (BYOD) to promote student learning. Interactive Learning Environments. 25(8), 983-995.

Karabulut-Ilgu, A., Jaramillo Cherrez, N. and Hassall, L. (2018). Flipping to engage students: Instructor perspectives on flipping large enrolment courses. Australasian Journal of Educational Technology. 34(4), 123-137.

Kerr, B. (2015). The flipped classroom in engineering education: A survey of the research. In Interactive Collaborative Learning (ICL), 2015 International Conference, Florence, Italy, 815-818.

Lage, M. J., Platt, G. J., \& Treglia, M. (2000). Inverting the classroom: A gateway to creating an inclusive learning environment. Journal of Economic Education, 31, 30-43.

Mason, G. S., Shuman, T. R., \& Cook, K. E. (2013). Comparing the effectiveness of an inverted classroom to a traditional classroom in an upper-division engineering course. IEEE Transactions on Education, 56(4), 430-435.

McClelland, C. J. (2013). Flipping a Large-enrollment Fluid Mechanics Course-Is it Effective?. In Proceedings of the 200th ASEE Annual Conference \& Exposition. Atlanta, GA.

Papadopoulos, C. \& Santiago-Román, A. (2010). Implementing an inverted classroom model in engineering statics: Initial results. Paper presented at the Annual Conference

\section{REFERENCIAS}


of the American Society for Engineering Education. PLACE.

Sanchidrián, J. A. (2014). Transferencia de Calor. $2^{\mathrm{o}}$ ed, Fundación Gómez-Pardo, E.T.S.I. Minas y Energía Universidad Politécnica de Madrid.

Velegol, S. B., Zappe, S. E., \& Mahoney, E. (2015). The Evolution of a Flipped Classroom: Evidence-Based
Recommendations. Advances in Engineering Education, 4(3), 1-37.

Yildirim, S., Bölen, M. \& Yildirim, G. (2017). Learners' views about cloud computing-based group activities. SHS Web of Conferences, 37, 10-33. 